\title{
Scale-free networks in complex systems
}

\author{
M. Bartolozzi ${ }^{a, b}$, D.B. Leinweber ${ }^{a, b}$, T. Surungan ${ }^{a, b, c}$, A.W. Thomas ${ }^{a, b, d}$ and A.G. Williams ${ }^{a, b}$ \\ ${ }^{a}$ Special Research Centre for the Subatomic Structure of Matter (CSSM), University of \\ Adelaide, Adelaide, SA 5005, Australia \\ ${ }^{b}$ Department of Physics, University of Adelaide, Adelaide, SA 5005, Australia \\ ${ }^{c}$ Department of Physics, Hasanuddin University, Makassar 90245, Indonesia \\ ${ }^{d}$ Jefferson Laboratory, 12000 Jefferson Ave., Newport News, VA 23606, USA
}

\begin{abstract}
In the past few years, several studies have explored the topology of interactions in different complex systems. Areas of investigation span from biology to engineering, physics and the social sciences. Although having different microscopic dynamics, the results demonstrate that most systems under consideration tend to self-organize into structures that share common features. In particular, the networks of interaction are characterized by a power law distribution, $P(k) \sim k^{-\alpha}$, in the number of connections per node, $k$, over several orders of magnitude. Networks that fulfill this propriety of scale-invariance are referred to as "scale-free". In the present work we explore the implication of scale-free topologies in the antiferromagnetic (AF) Ising model and in a stochastic model of opinion formation. In the first case we show that the implicit disorder and frustration lead to a spinglass phase transition not observed for the AF Ising model on standard lattices. We further illustrate that the opinion formation model produces a coherent, turbulent-like dynamics for a certain range of parameters. The influence, of random or targeted exclusion of nodes is studied.
\end{abstract}

Keywords: Scale-free networks, spin glass, complex systems, sociophysics

\section{INTRODUCTION}

Systems composed of many parts that interact with each other in a non-trivial way are often referred to as complex systems. An intriguing issue concerns the role played by the topological structures in the dynamics of these systems. Recent empirical studies focusing on the properties of interactions in different biological, social and technological systems have made it possible to shed some light on the basic principles of structural selforganization. A few examples include food webs, ${ }^{1-3}$ power grids and neural networks, ${ }^{4,5}$ cellular networks, ${ }^{6,7}$ sexual contacts, ${ }^{8}$ Internet routers, ${ }^{9-11}$ the World Wide Web, ${ }^{12,13}$ actor collaborations, ${ }^{4,5,12,14}$ the citation networks of scientists ${ }^{15,16}$ and the stock market. ${ }^{17,18}$ Although different in the underlying interaction dynamics or micro-physics, most of the empirical studies have shown a tendency of the networks to self-organize in structures that share common features. In particular, the number of connections, $k$, for each element, or node, of the network follow a power law distribution, $P(k) \sim k^{-\alpha}$. Networks that fulfill this property are referred to as scale-free networks (SFN). In addition many of these networks are characterized by a high clustering coefficient, $C$, in comparison with random graphs. ${ }^{19}$ The clustering coefficient, $C$, is computed as the average of local clustering, $C_{i}=2 y_{i} /\left(z_{i}\left(z_{i}-1\right)\right)$, where $z_{i}$ is the total number of nodes linked to the site $i$ and $y_{i}$ is the total number of links between those nodes. As a consequence both $C_{i}$ and $C$ lie in the interval $[0,1]$. The high level of clustering found, supports the idea that a herding phenomenon is a common feature in social and biological communities. Numerical studies on SFNs have demonstrated how the topology plays a fundamental role in infection spreading ${ }^{10}$ and tolerance against random and preferential node removal. ${ }^{20-22}$ A detailed description of the progress in this emerging field of statistical mechanics can be found in recent reviews. ${ }^{23,24}$

In the following section we briefly describe the algorithm used for generating the SFN, that is the BarabásiAlbert model with tunable clustering. We then study the implications of a SFN topology in two different complex systems. The first one, Sec. 3, in the antiferromagnetic (AF) Ising model while the second, Sec. 4, is a stochastic model for opinion formation.

Marco Bartolozzi: E-mail: mbartolo@physics.adelaide.edu.au 


\section{THE BARABÁSI-ALBERT MODEL WITH TUNABLE CLUSTERING}

The Barabási-Albert model ${ }^{12}$ is based on two main assumptions: (i) linear growth and (ii) preferential attachment. In practice the network is initialized with $m_{0}$ disconnected nodes. At each step a new node with $m$ edges is added to the pre-existing network. The probability that an edge of the new node is linked with the $i$ th node is expressed by $\Pi\left(k_{i}\right)=k_{i} / \sum_{j} k_{j}$. The iteration of this preferential growing process yields a scale free network, $P(k) \sim k^{-\alpha}$ where the degree distribution parameter $\alpha=3$.

It is worth noting that the Barabási-Albert model cannot produce a high clustering coefficient. In fact, the value of this coefficient depends on the total number of nodes, $N$, in the network ${ }^{23}$ and in the thermodynamic limit, $N \rightarrow \infty, C \rightarrow 0$. In principle the observed local clustering can play an important role in the opinion formation of groups of people, independent of their total number. In order to account for this, we introduce a further step in the growth process, namely the triad formation proposed by Holme and Kim. ${ }^{25}$ In this case, if the new added node is linked with an older node, $i$, having other links, then with a certain probability, $\theta$, the next link of the new node, if any remain, will be added to a randomly selected neighbour of node $i$. This method of introducing friends to friends, while preserving the scale-free nature of the networks, generates high clustering coefficients that do not depend on the number of nodes in the network. The only tunable parameter that changes the value of the clustering coefficient is the clustering probability $\theta$. All the simulations in the present work have been carried out using $\theta=0.9$, providing to an average clustering coefficient of $C \sim 0.39$, close to the value found in many real systems. ${ }^{23}$

\section{SPIN-GLASS BEHAVIOUR OF THE ANTIFERROMAGNETIC ISING MODEL ON SCALE-FREE NETWORK}

The ubiquity of SFNs in nature has inspired physicists to investigate the dynamics of standard models in the new case where the interactions between elements are described by complex interactions. These include the study of various magnetic models such as the Ising model. An intriguing issue concerns how the unusual topology acts to influence the cooperative behaviour of the spins. Studies of the ferromagnetic (FM) Ising model on a SFN, using several theoretical techniques ${ }^{26-29}$ including the Monte Carlo (MC) method, ${ }^{29}$ have found the robustness of ferromagnetic ordering against thermal fluctuations for the degree distribution exponent $\alpha \leq 3$.

The robustness feature is naturally expected as SFNs have large connectivities. This is analogous to the FM Ising model on a regular lattice above the lower critical spatial dimension, $d_{l}=2$. There the ordered phase is very robust against thermal fluctuations. However, for the antiferromagnetic (AF) case with a SFN, the situation is different.

Two factors come to play a central role in the dynamics of the AF-SFN model; namely the competition induced by the AF interaction in the elementary triangles of the network and the randomness related to the non-regular connections. The abundance of elementary triangles in the network leads to frustration, as, for example, only two of the three spins can be anti-aligned. More generally, frustration refers to the inability of the system to remain in a single lowest energy state (ground state). These ingredients lead the AF SFN to belong to a class of randomly frustrated systems commonly referred to as spin glasses (SGs).

Most studies of SGs have been performed on regular lattices. These studies have shown that frustration and randomness are the key ingredients for SG behavior, characterized by a frozen random spin orientation at low temperatures. ${ }^{30}$ A study of the AF Ising model on a SFN is of great theoretical interest since, in fact, it does possess all the characteristics of a SG. Reviews on SG can be found in Refs.. ${ }^{30}$

We consider the AF Ising model on a Barabási-Albert network with a tunable clustering coefficient, as described in Sec. 2. We illustrate that the AF model undergoes a SG transition. Such a transition is not observed on a regular triangular lattice where, for the AF Ising model, the spins are fully frustrated.

\subsection{Model and Simulation Method}

On each SFN constructed at the beginning of the simulation, we assign to each vertex an Ising spin, and to each link an AF interaction. The Hamiltonian can be written as follows

$$
H=-\sum_{\langle i j\rangle} J_{i j} s_{i} s_{j}
$$


Here the summation is performed over the connected spins $s_{i}$ and $s_{j}$ occupying sites $i$ and $j$, respectively. The coupling interaction $J_{i j}=J<0$ is AF. As previously mentioned, each vertex with the local cluster coefficient $C_{i}>0$ together with its neighbours, compose elementary triangles. Due to the AF interactions the local system is frustrated.

It is worth pointing out that $C$ is related to the degree of frustration of each network. Due to the probabilistic algorithm used for their construction, the value of $C$ fluctuates from one network to the next. This property is not shared by other algorithms which use recursion formulas to generate scale-free structures, such as, for example, the Apollonian networks. ${ }^{31}$

As a random system, each realization of a network of size $N$ will differ in the "structure" of connectivities. Therefore, in order to have reliable statistics, we average over many realizations of the SF network for each specified size. In general, one takes into account more realizations for small system sizes and less for large system sizes as the latter tend to self-average. The system sizes that we simulate are $N=1024,2048,4096$, and 8192. Since the self-averaging of physical quantities for larger system sizes are interfered by the increase of ground state degeneracy, we did not take less realizations. Instead all physical quantities of interest for each system size are averaged over 1000 network realizations.

Another peculiarity of SF networks regards the existence of a broad distribution of "hubs", that is nodes with a large number of connections, $k$. The energy difference in a spin flip actually depends on the number of connections of the spin itself, $\Delta E_{i}=-2 s_{i} \sum_{j=1}^{k_{i}} s_{j}$. Thus in the AF case for the $i$ th spin with $k_{i}$ connections, the hubs are more likely to "freeze" into a particular configuration compared to the nodes with just few links. This fact resembles the spin glass behaviour of particular alloys where some elements freeze into a particular orientation at a higher temperature than others.

The calculation of the thermal averages of the physical quantities of interest is performed using the replica exchange MC method, ${ }^{32}$ appropriate for systems such as spin-glass. For a given network configuration, replicas having an associated inverse temperature, $\beta$, are created. In using this method, we define a "local" MC (LMC) update as a MC update for each spin of each replica, either consecutively through all elements of the network or randomly. Given that we can group the inverse temperatures in even and odd pairs, $\left(\beta_{m}, \beta_{m+1}\right)$, after each LMC update we alternate attempts to switch configurations from one temperature to the next. According to this procedure, we define a Monte Carlo step (MCS) as a LMC plus a half ( $m$ odd or even) exchange trial.

For each network realization we run $3 \times 10^{5}$ MCSs after a transient period of $10^{3}$ LMC updates. We take a total of $6 \times 10^{4}$ measures for the thermal averages. The simulation is run down to low temperatures in a search for the possible existence of a phase transition. All the thermal averages obtained are then averaged over the whole ensemble of networks. In the following, we indicate $\langle\ldots\rangle$ as the thermal average and $[\ldots]_{a v}$ as the ensemble average. The statistical errors in the plots, where reported, are calculated via the bootstrap method.

\subsection{Observing Spin Glass Behaviour}

With the presence of frustration and randomness in the AF-SFN model, we expect to observe a spin glass transition, i.e., a transition from a temporal disordered to a temporal ordered phase at low temperatures. A quantity that is often used to characterize the SG state is the overlap parameter, $q$, defined as ${ }^{33}$

$$
q=\left[\left\langle\frac{1}{N} \sum_{i} s_{i}^{\alpha} s_{i}^{\beta}\right\rangle\right]_{a v},
$$

where the superscripts $\alpha$ and $\beta$ denote two copies of the same configuration of connectivity at the same temperature.

In particular, for the Ising system, due to the $Z_{2}$ symmetry, it is important to evaluate the absolute value of the order parameter, $|q|=\left[\left\langle\left|1 / N \sum_{i} s_{i}^{\alpha} s_{i}^{\beta}\right|\right\rangle\right]_{a v}$, to overcome the implication of the $Z_{2}$ symmetry of the Hamiltonian. That is, if the system is at thermal equilibrium and if we take quite long MCS then the usual $q$ should average out and give an approximately zero value. The existence of a spin glass phase is indicated by the convergence of $|q|$ to a finite value as we increase the network size and, at the same time, a convergence of $|q|$ to zero at high temperatures. In the latter case the system is in the paramagnetic phase. 

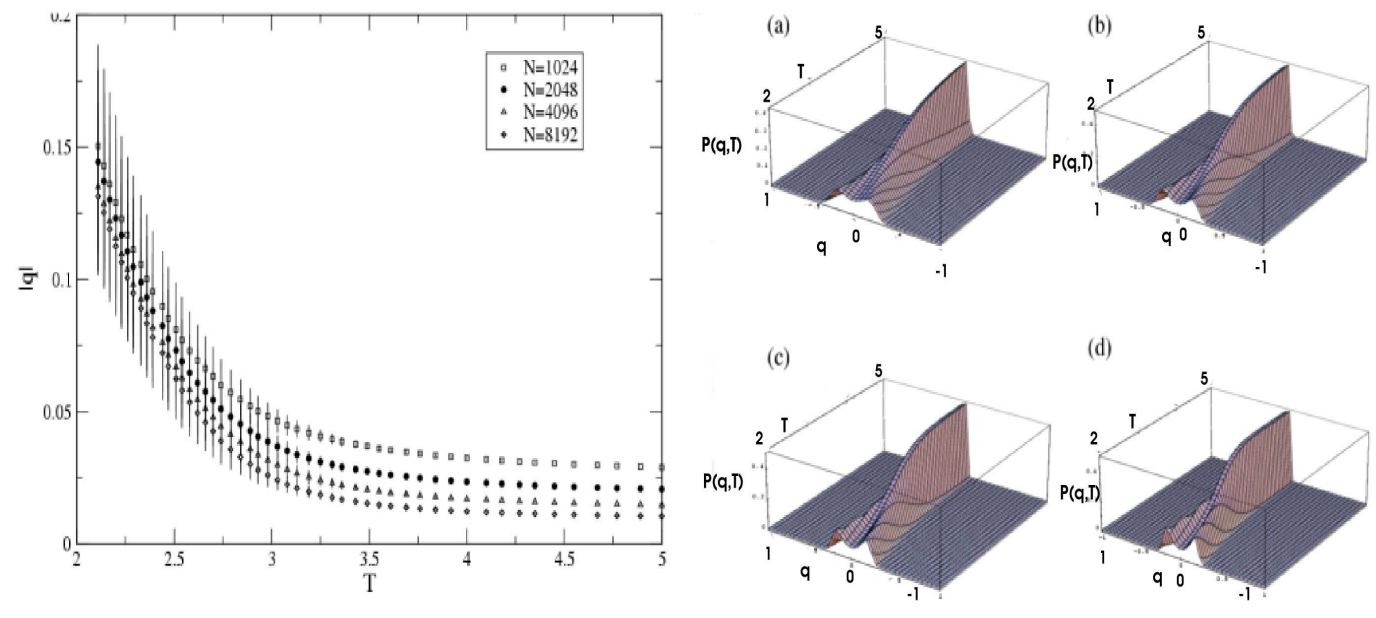

Figure 1. Left: Temperature dependence of the overlap parameter, $q$, for different system sizes $N$. The increasing value of $q$ at low temperatures indicates a SG phase. For a given network size, 1000 realizations of the SFN are averaged over. Right: The distribution of $q$ at various temperatures for different system sizes, including (a) $N=1024$, (b) $N=2048$, (c) $N=4096$ and (d) $N=8192$. The temperatures are provided in units of $J / k_{B}$, where $k_{B}$ is the Boltzmann constant.

The temperature dependence of $|q|$, resulting from the simulations, is shown in Fig. 1 (Left). The existence of a SG phase is indicated by the finite value of $|q|$ in the low temperature region, and the approach of $|q|$ to zero at higher temperatures associated with paramagnetic phase. For high temperatures and large networks, $|q|$ is approaching zero in accord with the thermodynamic limit where $|q|=0$.

The existence of these two different phases can also be observed from the distributions of $q$, as shown in Fig. 1 (Right). For higher temperatures we observe simple Brownian fluctuations of the values of $q$, leading to a singly peaked Gaussian distribution characteristic of a paramagnetic state. By decreasing the temperature, the distribution starts to spread out, reflecting the increasing number of metastable disordered states reflecting the presence of substantial frustration. At lower temperatures the distribution develops double peaks associated with the Edward-Anderson parameter representative of the SG phase. The transition between these two phases is roughly estimated at $T \sim 4$. We note that the shape of the observed distribution is different from that of the conventional Ising system where the double peaks approach delta-like double peaks reflecting a simple doubly degenerate ground state.

A more accurate evaluation of the phase transition is done through the Binder parameter defined as follows

$$
g_{L}=\frac{1}{2}\left(3-\frac{\left[\left\langle q^{4}\right\rangle\right]_{a v}}{\left[\left\langle q^{2}\right\rangle\right]_{a v}^{2}}\right),
$$

where $\left\langle q^{2}\right\rangle$ and $\left\langle q^{4}\right\rangle$ are respectively the second and the fourth cumulant moment of $q$ and $0 \leq g_{L} \leq 1$. At high temperature, when the thermal fluctuation overcomes all cooperative interaction, the system is expected to exist in the paramagnetic phase where there is no spatial nor temporal autocorrelation. As a result, the distribution of $q$ should be Gaussian centered at $q=0$. In this case the ratio of the cumulants, $\left\langle q^{4}\right\rangle /\left\langle q^{2}\right\rangle^{2} \rightarrow 3$, resulting in $g_{L} \rightarrow 0$. At low temperatures, the cooperative interaction becomes dominant and the ratio of the cumulants approaches unity so that $g_{L}=1$.

Fig. 2 (Top) displays the temperature dependence of the Binder parameter for a variety of network sizes. A spin glass state is observed for lower temperatures where the Binder parameter deviates from zero, and increases with the system size. In the thermodynamic limit, we expect $g_{L} \rightarrow 1$ just below the critical temperature. A crossing point in the size dependence of $g_{L}$ indicates that the critical temperature for the SG phase transition 


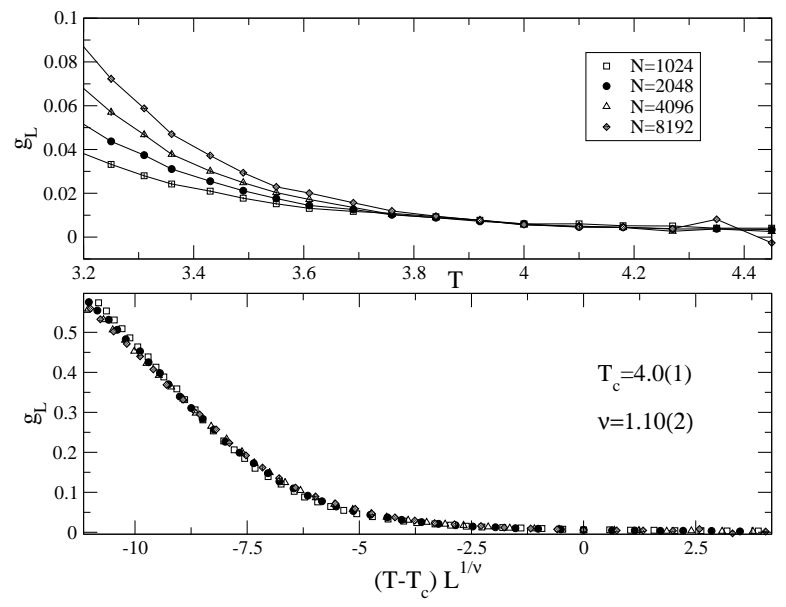

Figure 2. Top: Scaling behaviour of the Binder cumulant, $g_{L}$, for different system sizes. Each system size is averaged over 1000 realizations of the network configuration. Bottom: Scaling plot of the data illustrated above (Top), fit to Eq. 4 .

is $T \sim 4.0$. For temperatures above $T \sim 4.0$ the Binder parameter, while remaining always above zero, does indeed order in an opposite manner indicative of a genuine crossing of the curves and in accord with a genuine spin glass transition at finite temperature.

A more reliable estimate of the critical temperature, $T_{c}$, for finite size systems can be given by using scaling arguments. For a SG system, the Binder parameter depends on the system size $L$ as

$$
g_{L}=\tilde{g}_{L}\left[\left(T-T_{c}\right) L^{1 / \nu}\right]
$$

with $\nu>0$. At $T_{c}$ the Binder cumulant does not depend on $L$. For the SFN, the system size scales logarithmically with the number of nodes $N,{ }^{23}$ and therefore we take $L=\log (N)$. The parameters $T_{c}$ and $\nu$ are determined by constraining the temperature dependence of the Binder parameter for each network size to lie on a single curve. The curves following the scaling bahaviour of Eq. (4) are shown in Fig. 2 (Bottom). ¿From this fit we estimate the critical temperature $T_{c} \sim 4.0(1)$ and the exponent of the SG correlation length $\nu \sim 1.10(2)$. It is important to underline that this kind of behaviour is not observed for an AF system on a regular triangular lattice.

\section{STOCHASTIC MODEL OF OPINION FORMATION ON A SCALE-FREE NETWORK}

We turn now our attention to the role played by the SFN on a model of stochastic opinion formation. In this case, once the scale-free network has been built, we randomly assign the spin values, \pm 1 , to every node. These values correspond to a Boolean kind of opinion while the bonds of the networks represent the interactions between agents.

The dynamics of the spins follows a stochastic process that mimics the human uncertainty in decision making. ${ }^{34,35}$ Values are updated synchronously according to a local probabilistic rule: $\sigma_{i}(t+1)=+1$ with probability $p_{i}$ and $\sigma_{i}(t+1)=-1$ with probability $1-p_{i}$. The probability $p_{i}$ is determined, by analogy with heat bath dynamics with formal temperature $k_{B} T=1, p_{i}(t)=1 /\left(1+e^{-2 I_{i}(t)}\right)$, where the local field, $I_{i}(t)$, is

$$
I_{i}(t)=a \xi(t) \tilde{N}_{i}^{-1} \sum_{j=1}^{\tilde{N}_{i}} \sigma_{j}(t)+h_{i} \eta_{i}(t) r(t) .
$$



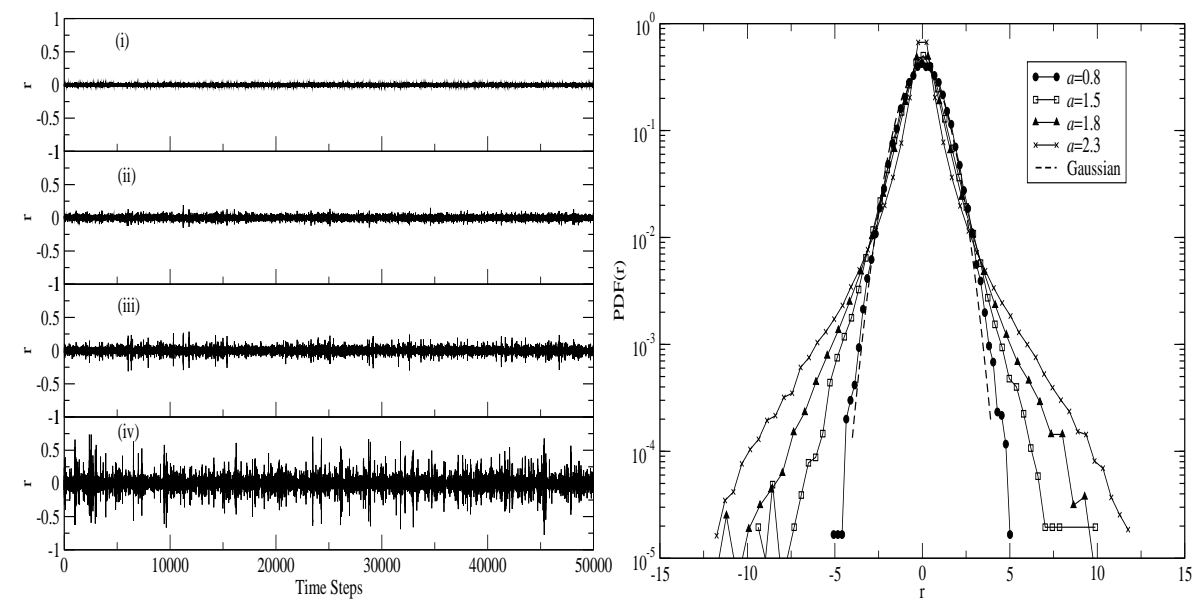

Figure 3. Left: Time series of the average opinion, $r$, for different values of the group interaction strength parameter $a$ : (i) $a=0.8$, (ii) $a=1.5$, (iii) $a=1.8$ and (iv) $a=2.3$. The parameters used for the simulations are: $N=10^{4}$ nodes, clustering probability $\theta=0.9$, initial nodes and links per new node $m_{0}=m=5$ and we take the upper bound of the distribution of personal response strengths equal to the group interaction strength, that is $\kappa=a$. The results involve 10 realizations of the scale free network each displayed for 5000 time steps. For values of $a$ greater than 1 a turbulent-like state, characterized by large fluctuations, starts to appear in the process of opinion formation. Right: PDFs of the time series relative to the time series in (Left). The shapes of the distributions converge to a Gaussian for small values of the group interaction strength $a=\kappa$. A Gaussian distribution is also plotted for comparison. All the PDFs in this paper are obtained over 50 realizations of the SF network. In order to compare the fluctuations at different scales, the time series in the plot have been normalized according to $r(t) \rightarrow \frac{r(t)-\bar{r}}{\sigma}$, where $\bar{r}$ and $\sigma$ denote the average and the standard deviation over the period considered respectively.

The first term on the right-hand side of Eq. (5) represents the time dependent interaction strengths between the node $i$ and his/her $\tilde{N}_{i}$ information sources, which are the first neighbours in the network. The second term instead reflects the personal reaction to the system feedback, that is the average opinion, $r(t)=1 / N \sum_{j=1}^{N} \sigma_{j}(t)$, resulting from the previous time step. The terms $\xi(t)$ and $\eta_{i}(t)$ are random variables uniformly distributed in the interval $(-1,1)$ with no correlation in time nor in the network. They represent the conviction, at time $t$, with which agent $i$ responds to his/her group (common for all the agents) and the global opinion of the network respectively. The strength term, $a$, is constant and common for the whole network, while $h_{i}$ is specifically chosen for every individual from a uniform distribution in $(0, \kappa)$ and are both constant in the dynamics of the system. By varying the parameter $\kappa$ we can give more or less weight to the role of feedback in the model. The strength coefficients $a$ and $h_{i}$ in the local field, $I_{i}$, characterizing the attributes of the agents, play a key role in the dynamics of the model. They represent the relative importance that each agent of the network gives, respectively, to his/her group and to the variation of the average opinion itself. While $a$ is a parameter associated with the network, $h_{i}$ is specifically chosen for each individual at the beginning of each simulation.

\subsection{Numerical Simulations}

At first we investigate the importance of the group strength $a$ by fixing $\kappa=a$. In this case the dynamical behaviour is similar to that found in the stock market context in Refs.. ${ }^{34-36}$ For $a \tilde{<} 1$ the resulting time series of average opinion is largely uncorrelated Gaussian noise with no particularly interesting features, as illustrated in Fig. 3(i) (Left).

As soon as we exceed the value of $a \approx 1$ a turbulent-like regime sets in, characterized by large intermittent fluctuations, Fig. 3(ii $\rightarrow$ iv) (Left). These large fluctuations, or coherent events, can be interpreted in terms of a multiplicative stochastic process with a weak additive noise background. ${ }^{34,37}$ For $a>2.7$ we observe that the bursts of the time series begin to saturate the bounds $-1 \leq r \leq 1$. 
In Fig. 3 (Right) we plot the probability distribution functions (PDFs) associated with the time series of Fig. 3 (Left). The large fluctuations, for $a$ greater than $\approx 1$, are reflected in the fat tails of the relative PDFs. Decreasing the value of $a$, and so the number of coherent events, the PDF converges to a Gaussian distribution generated by a random Poisson process.

In order to test the relevance of the network structure on the process of opinion formation, the previous simulations have been repeated, with a large number of nodes, $N$, and $\kappa=a$, for different values of the clustering parameter, $\theta$, and the node-edge parameter, $m$. While varying $\theta$, does not lead to any substantial difference in the dynamics of the model, the increase of the average number of links per node, $\bar{k}=2 \mathrm{~m}$, has a dramatic effect in the turbulent-like phase, which deviations from a Gaussian regime increase dramatically: large scale synchronizations are more likely to occur for large $m$. This behaviour is intrinsically related to the model of Eq. (5). In fact, the turbulent-like regime is a consequence of the random fluctuations of the interaction strengths between agents around a bifurcation value separating the ordered and disordered phase.

It is also worth pointing out that an increase of $\bar{k}$ is related to a decrease in the average path length between nodes; that is, the network "shrinks" and becomes more compact. In relation to our previous discussion, the more compact the network is the more the dynamics of our system approaches to the mean field approximation. It becomes easier for the agents to synchronize. This characteristic of compactness, referred to as the small world effect, ${ }^{19,23,24}$ is actually very common in both real and artificial networks.

These results confirm that the critical topological characteristic leading to herding behaviour in the framework of stochastic opinion formation is the presence of mean field effects enhanced by small-world structure. The more information (links) that an agent has, the more likely it is for him/her to have an opinion in accord with other agents.

\subsection{The Influence of Indecision}

We now extend our model in order to include the concept of indecision. In practice a certain agent $i$, at a time step $t$, may take neither of the two possible decisions, $\sigma_{i}= \pm 1$, but remain in a neutral state. Keeping faith to the spirit of the model, we address this problem introducing an indecision probability, $\epsilon$ : that is the probability to find, at each time step, a certain agent undecided. This is equivalent to introducing time dependent failures in the structure of the network by setting $\sigma=0$.

Focusing on the turbulent-like regime, the shape of the PDF in the opinion fluctuations changes according to different concentrations of undecided persons. The results of the simulations, in Fig. 4 (Left), show how the dynamics of the model move from an intermittent state for $\epsilon=0$ toward a noise state for $\epsilon \approx 0.6$. The convergence to a Gaussian distribution is obtained only for quite high concentrations of undecided agents at about $60 \%$. The robustness of the turbulent-like behaviour is related to the intrinsic robustness of SF networks against random failures. ${ }^{20-22}$ In fact, because there is a large absolute number of poorly connected nodes, related to the power law shape of $P(k)$, the probability of setting one of them to inactive is much higher compared to the "hubs" that are relatively rare.

We can claim that, in large social networks governed by stochastic reactions in their elements, large fluctuations in the average opinion can appear even in the case in which a large part of the network is actually "inactive" provided that the structure is scale free and the indecision is randomly distributed. The existence of large hubs provides for the survival of extended sub-networks in which synchronization can give rise to coherent events. The structure of the network itself supplies the random indecision.

Now we address the question of how the dynamics may change if we do not choose randomly the inactive nodes but we target the nodes having the most links. What we do in practice is to sort the nodes according to their number of links and then deactivate the nodes having the largest number of links in decreasing order. Fig. 4 (Right) illustrates how the fragmentation process is much faster and the noise regime is reached already when only the $10 \%$ of the hubs are deactivated. As emphasized in Ref., ${ }^{20-22}$ the hubs have a great importance in the structural properties of SF networks and specifically targeting these nodes can lead to sudden isolation of a large fraction of the nodes of the network. 

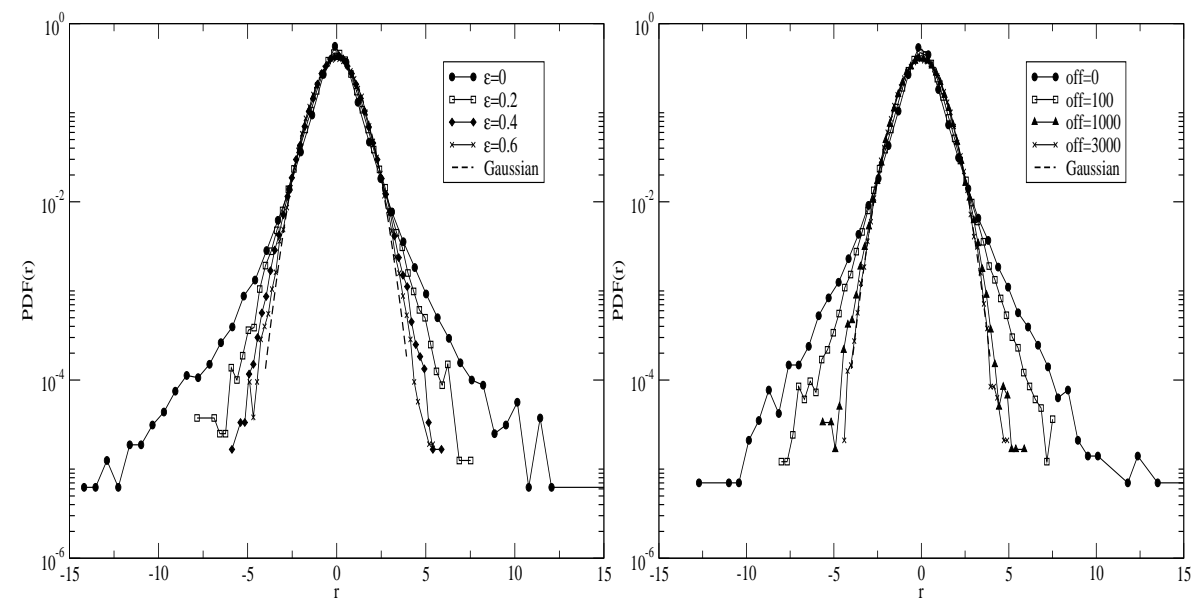

Figure 4. Left: Transition from coherent bahaviour, indecision probability $\epsilon=0$, to noise using a random selection for the inactive agents. For $\epsilon \approx 0.6$ we reach a noise-like behaviour. The parameters used in the simulation are: $N=10^{4}$ nodes, $\theta=0.9$ for the clustering probability, $m=m_{0}=5$ for the links of each new node, $a=1.8$ and $\kappa=a$ for the group and global opinion response respectively. Right: In this simulation we progressively turn off the largest hubs in the network. Once we have turned off about the $10 \%$ of agents, $N=10^{4}$, the coherence in opinion formation disappears.

\section{DISCUSSIONS AND CONCLUSIONS}

Motivated by recent empirical findings for complex systems interaction topologies, we have explored the implications of a Barabási-Albert SFN topology in two different models of complex systems. In particular we have found that the random frustration introduced by this topology of interactions induces a transition from a paramagnetic state to a spin glass state for an AF Ising model at a finite temperature. The critical spin glass phase transition temperature is estimated to be $T_{c} \sim 4.0(1)$. Such behaviour is not observed for the AF Ising model on regular lattices.

The SFN topology also has important consequences in our model for opinion formation. In this case, we discovered that the "hubs" of the network are more likely to synchronize due to mean field effects. Conversely, these effects are not strong enough to synchronize the poorly connected nodes. Moreover, introducing inactive agents and spreading them randomly on the network, does not spoil the turbulent-like state, even for high concentrations of "gaps" up to approximately $60 \%$ of agents. This is a consequence of the implicit robustness of $\mathrm{SF}$ networks against random failures. If instead of selecting randomly the undecided individuals, we aim directly to the "hubs" of the network then the situation changes. In this case the network is disaggregate, composed of very small sub-networks and isolated nodes. Synchronization cannot significantly effect the resulting global opinion and the time series approximates Gaussian noise.

\section{ACKNOWLEDGMENTS}

Part of the computation of this work has been done using super computer facilities of the South Australian Partnership for Advanced Computing (SAPAC).

\section{REFERENCES}

1. R. Williams and et al. Proc. Natl. Acad. Sci. U.S.A. 99, p. 12913, 2002.

2. J. Camacho, R. Guimera, and L. Amaral Phys. Rev. Lett. 88, p. 228102, 2002.

3. J. Montoya and R. Sole J. Theor. Biol. 214, p. 405, 2002.

4. D. Watts and S. Strogatz Nature (London) 393, p. 440, 1998.

5. L. Amaral and et al. Proc. Natl. Acad. Sci. U.S.A. 97, p. 11149, 2000.

6. H. Jeong and et al. Nature (London) 407, p. 651, 2000. 
7. H. Jeong and et al. Nature (London) 411, p. 41, 2001.

8. F. Liljeros and et al. Nature (London) 411, p. 907, 2001.

9. M. Faloutsos, P. Faloutsos, and C. Faloutsos Comp. Commun. Rev. 29, p. 251, 1999.

10. R. Pastor-Satorras and A. Vespignani Phys. Rev. Lett. 86, p. 3200, 2001.

11. S. Yook, H. Jeong, and A.-L. Barabási Proc. Natl. Acad. Sci. U.S.A. 99, p. 13382, 2002.

12. R. Albert, H. Jeong, and A.-L. Barabási Nature 401, p. 130, 1999.

13. R. Kumar and et al. Proceeding of the 19th ACM Symposium on Principles of Database systems (unpublished) , p. $1,2000$.

14. A.-L. Barabási and R. Albert Science 286, p. 509, 1999.

15. S. Redner Eur. Phys. J. B 4, p. 131, 1998.

16. A. Vazquez Europhys. Lett. 54, p. 430, 2001.

17. G. Bonanno, G. Caldarelli, F. Lillo, and R. Mantegna Phys. Rev. E 68, p. 046130, 2003.

18. J.-P. Onnela, A. Chakraborti, K. Kaski, J. Kertesz, and A. Kanto Phys. Rev. E 68, p. 056110, 2003.

19. B. Bollobás, Random graphs, Academic, London, 1985.

20. R. Albert, H. Jeong, and A.-L. Barabási Nature 406, p. 378, 2000.

21. R. Cohen, K. Erez, D. ben Avraham, and S. Havlin Phys. Rev. Lett. 85, p. 4626, 2000.

22. D. Callaway, M. Newman, S. Strogatz, and D. Watts Phys. Rev. Lett. 85, p. 5468, 2000.

23. R. Albert and A.-L. Barabasi Rev. Mod. Phys. 74, p. 47, 2002.

24. S. Dorogovtsev and J. Mendes Adv. Phys. 51, p. 1079, 2002.

25. P. Holme and B. Kim Phys. Rev. E 65, p. 026107, 2002.

26. A. Aleksiejuk, J. Holyst, and D. Stauffer Physica A 310, p. 260, 2002.

27. S. Dogorovtsev, A. Goltsev, and J. Mendes Phys. Rev. E 66, p. 016104, 2002.

28. F. Iglói and L. Turban Phys. Rev. E 66, p. 036140, 2002.

29. C. Herrero Phys. Rev. E 69, p. 067109, 2004.

30. K. Binder and A. Young Rev. Mod. Phys. 58, p. 801, 1986.

31. R. Andrade and H. Herrmann Phys. Rev. E 71, p. 056131, 2005.

32. K. Hukushima and K. Nemoto J. Phys. Soc. Japan 65, p. 1863, 1996.

33. G. Parisi Phys. Rev. Lett. 50, p. 1946, 1983.

34. A. Krawiecki, J. Holyst, and D. Helbing Phys. Rev. Lett. 89, p. 158701, 2002.

35. M. Bartolozzi and A. Thomas Phys. Rev. E 69, p. 046112, 2004.

36. T. Kaizoji Physica A 287, p. 493, 2000.

37. H. Nakao Phys. Rev. E 58, p. 1591, 1998. 